\begin{tabular}{|c|}
\hline $\begin{array}{r}\text { PRAMANA } \\
\text { - journal of } \\
\text { physics }\end{array}$ \\
\hline
\end{tabular}

\title{
Building better oscillators using nonlinear dynamics and pattern formation
}

\author{
$\mathrm{M} \mathrm{C} \mathrm{CROSS}^{1, *}$, EYAL KENIG ${ }^{1}$ and JOHN-MARK A ALLEN ${ }^{2}$ \\ ${ }^{1}$ Department of Physics, California Institute of Technology, Pasadena, California 91125, USA \\ ${ }^{2}$ Department of Computer Science, University of Oxford, Wolfson Building, Parks Road, Oxford, \\ OX1 3QD, UK \\ *Corresponding author. E-mail: mcc@ caltech.edu
}

DOI: $10.1007 / \mathrm{s}$ 12043-014-0924-1; $\boldsymbol{e P u b l i c a t i o n : ~} 18$ February 2015

\begin{abstract}
Frequency and time references play an essential role in modern technology and in living systems. The precision of self-sustained oscillations is limited by the effects of noise, which becomes evermore important as the sizes of the devices become smaller. In this paper, we review our recent theoretical results on using nonlinear dynamics and pattern formation to reduce the effects of noise and improve the frequency precision of oscillators, with particular reference to ongoing experiments on oscillators based on nanomechanical resonators. We discuss using resonator nonlinearity, novel oscillator architectures and the synchronization of arrays of oscillators, to improve the frequency precision.
\end{abstract}

Keywords. Oscillator; clock; nonlinear; noise; nanomechanics; synchronization.

PACS Nos 05.45.-a; 85.85.+j; 62.25.-g; 47.52.+j

\section{Introduction}

Oscillators play crucial roles in much of modern technology, for example in timekeeping (quartz crystal watches), communication (frequency references for mixing down radio frequency signals), and sensors. They are also important in timekeeping in biological systems. A key characteristic is the intrinsic frequency precision of the device. One way of quantifying this is to use the spectral density of the output signal in the frequency domain: the narrower the peak, the more precisely can the frequency be defined. Improving this frequency precision is the fundamental issue, broadly common to all oscillators, considered in this paper. There are other important practical characteristics, including the robustness of the frequency to environmental perturbations such as vibrations and temperature fluctuations and long term drift due to aging, that are more dependent on the details of the device implementation; these are not considered here. 


\section{C Cross, Eyal Kenig and John-Mark A Allen}

Unlike a resonator driven by an external oscillating signal, where the linewidth of the spectral response as the frequency is swept is determined by the dissipation in the resonator, the linewidth of a self-sustained oscillator is only nonzero in the presence of noise. An oscillator is mathematically described by a limit cycle in the phase space of dynamical variables, and the linewidth of the signal corresponding to a limit cycle is zero. Dissipation serves to relax the system to the limit cycle, which is itself determined by a balance of energy injection and dissipation, but does not broaden the spectral line. The spectral line is broadened only if there is some stochastic influence that causes the phase-space trajectory to fluctuate away from the limit cycle. Thus, the frequency imprecision (linewidth) is due to noise.

High-precision clocks and frequency references are typically based on a high-quality factor $(Q)$ resonator, often mechanical, used as the frequency defining element. A familiar example is the quartz crystal resonator in watches, cell phones, etc. Providing the resonator is driven at an amplitude such that nonlinear effects are small, the frequency is insensitive to the amplitude of the motion, and so imperfect control of this quantity does not degrade the frequency precision. This is the same principle described by Galileo three centuries ago. On the other hand, the effects of noise acting on the resonator are reduced by going to higher amplitudes. Thus, the limiting operating condition presently used to optimize performance is to drive the resonator as strongly as possible before nonlinearity degrades the performance too much. As attempts are made to reduce the size of the resonator, using MEMS or NEMS, the noise becomes relatively larger, and this strategy will eventually fail to give the desired performance. In this paper, we discuss theoretical work aimed to mitigate the bad effects of resonator nonlinearity on oscillator performance and to exploit the nonlinearity in novel ways to improve the performance. Our focus is on oscillators built from nanomechanical devices, but the ideas apply generally. This paper is a summary of work published in a number of papers [1-6]; more details can be found in those publications. There is also experimental work supporting some of the results $[7,8]$.

\section{Oscillator phase noise}

An oscillator on a limit cycle can be characterized by a phase variable $\theta$ roughly giving the angle around the limit cycle. It is convenient to perform a nonlinear transformation so that the phase variable advances uniformly in time and then the time derivative $\dot{\theta}=\omega$ is the frequency of the oscillations. The net effect of noise acting on the oscillator is that the advance of the phase becomes nonuniform, and the frequency precision is degraded. This idea has been studied for many decades: some early references are Lax [9], Kaertner [10], and Demir et al [11]. A key idea is that the phase is a free variable: any phase can be chosen as an initial condition, and a shift in the phase is equivalent to a time translation. A phase shift is therefore a zero (Goldstone) mode of the Floquet stability analysis of the limit cycle resulting from time translational symmetry.

There is no restoring force to correct a phase shift induced by a noise kick: as a result the phase undergoes Brownian motion leading to phase diffusion [12]. This phase diffusion leads to a broadening of the spectral peak and a degradation in the frequency precision. Noise kicks will also, in general, excite the exponentially decaying modes corresponding to the stable eigenvalues of the Floquet analysis. These deviations will shift 
weight from the spectral peak to Lorentzian sidebands with widths given by the decay rates, but, in the absence of phase diffusion, will leave the delta-function peak of the ideal limit cycle sharp (cf. the Debye-Waller factor of Bragg peaks for X-ray scattering off a crystal). Thus, the fundamental limits of frequency precision are determined by the phase diffusion.

The effect of a noise perturbation $\xi(t) \mathbf{v}_{n}(t)$ with $\mathbf{v}_{n}(t)$ giving the direction of the noise kick in the phase space, and $\left\langle\xi(t) \xi\left(t^{\prime}\right)\right\rangle=f \delta\left(t-t^{\prime}\right)$ for white noise, can be calculated via the solvability condition of secular perturbation theory [11]. The resulting phase diffusion depends on the scalar product of the noise vector $\mathbf{v}_{n}(t)$ with the phase sensitivity vector $\mathbf{v}_{\perp}(t)$ defined as the zero-eigenvalue adjoint eigenvector $\mathbf{e}_{0}^{\dagger}$ of Floquet stability analysis of the limit cycle, with the normalization $\mathbf{e}_{0}^{\dagger} \cdot \mathbf{e}_{0}=1$, where $\mathbf{e}_{0}$ is the vector corresponding to a unit phase shift of the oscillator, and is the zero-eigenvalue eigenvector of the stability analysis. The noise kicks projected along the phase direction then lead to Brownian motion of the phase and phase diffusion, so that in the long time limit

$$
\left\langle\left(\theta(t)-\omega_{0} t\right)^{2}\right\rangle \rightarrow 2 D t
$$

with

$$
D=f \overline{\left[\mathbf{v}_{\perp}(t) \cdot \mathbf{v}_{n}(t)\right]^{2}}
$$

where the bar denotes an average around the limit cycle and $\omega_{0}$ is the frequency of the oscillator, which may be changed from the no-noise frequency by a correction proportional to the noise strength. The correlation function of a periodic function of the phase giving the output signal, e.g., $x=\cos \theta(t)$, decays exponentially. For $t>0$

$$
\begin{aligned}
\langle x(t) x(0)\rangle & \propto \operatorname{Re}\left\langle\mathrm{e}^{i[\theta(t)-\theta(0)]}\right\rangle \\
& =\mathrm{e}^{-\left\langle\left[\theta(t)-\omega_{0} t\right]^{2}\right\rangle / 2} \cos \omega_{0} t=\mathrm{e}^{-D t} \cos \omega_{0} t .
\end{aligned}
$$

The corresponding spectral density (the Fourier transform of the correlation function) is a Lorentzian with width proportional to the noise strength $f$

$$
S_{x x}(\omega)=\frac{S_{0}}{\pi} \frac{D}{\left(\omega-\omega_{0}\right)^{2}+D^{2}} .
$$

Thus the linewidth giving the frequency imprecision is presented by calculating the phase diffusion coefficient $D$ (eq. (2)).

In the following sections, these basic ideas are applied to two important schemes to improve oscillator performance: first, improving a single oscillator using nonlinear dynamics of the component resonator; and secondly using synchronization of arrays of oscillators.

\section{Improving frequency precision using nonlinear dynamics}

A simple schematic of an oscillator based on a high- $Q$ mechanical resonator is shown in figure 1. The output signal from the resonator is amplified and fed back to drive the resonator with a phase shift to give positive feedback. Two types of noise sources can be anticipated: noise acting directly on the resonator, including the thermomechanical 


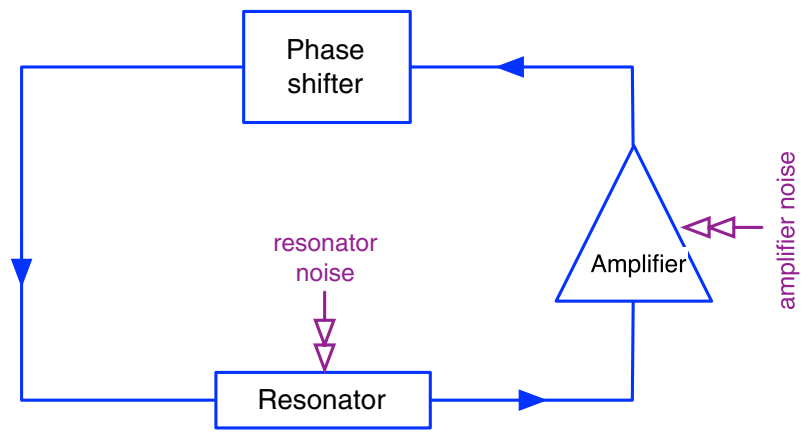

Figure 1. Schematic of a feedback oscillator.

noise associated with the resonator dissipation via the fluctuation-dissipation theorem, and parameter noise such as mass fluctuations due to molecules binding and unbinding with the device or spring constant fluctuations due to temperature fluctuations; and electronic noise from the amplifier/phase-shifter combination adding noise to the feedback drive.

The resonator is described by the equation of motion for the displacement $x(t)$ of the chosen mode of oscillation

$$
\ddot{x}+Q^{-1} \omega_{0} \dot{x}+\omega_{0}^{2} x+b x^{3}=d(t)
$$

where $\omega_{0}$ is the linear resonance frequency and $Q$ is the quality factor. The last term on the left-hand side gives the crucial nonlinearity we shall be discussing: a nonlinear spring stiffening or softening given by the coefficient $b$, often called the Duffing term [13]. The term on the right-hand side is the drive $d(t)$, which in the closed loop is the feedback.

The effect of nonlinearity on the open-loop periodically-driven resonator $d(t)=$ $d_{0} \cos \omega t$ is shown in figure 2 , and is often called the 'Duffing effect'. The gain $a_{0} / d_{0}$, with $x=a_{0} \cos (\omega t+\theta)$, normalized to the value on resonance $Q$, is plotted for three drive levels $d_{0}$ as a function of the scaled frequency offset $Q\left(\omega-\omega_{0}\right) / \omega_{0}$. Above a 'Duffing critical drive level' (middle panel), the resonance peak becomes re-entrant, giving multiple solutions over a range of drive frequencies due to the nonlinear frequency pulling. (The plot is for spring stiffening, $b>0$.)

The simple reasoning in designing a closed-loop oscillator is that the nonlinear frequency pulling adversely affects the performance, because the resonance frequency is now a function of amplitude. However, Greywall et al [14] and Yurke et al [15] showed that for the special case of a saturated amplifier, so that the feedback signal does not depend on the strength of the oscillations, there is in fact suppression of the effect of the feedback noise on the oscillator phase noise at the special oscillation amplitude given by the Duffing critical point. The effect of the direct resonator noise is actually worse under this condition than for the linear resonator, but if the feedback noise dominates, as is often the case, improved frequency precision is obtained. This is a rather surprising result, because one typically expects a larger effect of noise at a critical point. Also the relevance of the open-loop driven resonator curve to the closed loop oscillator is not immediately obvious. These aspects were studied in [4]. 

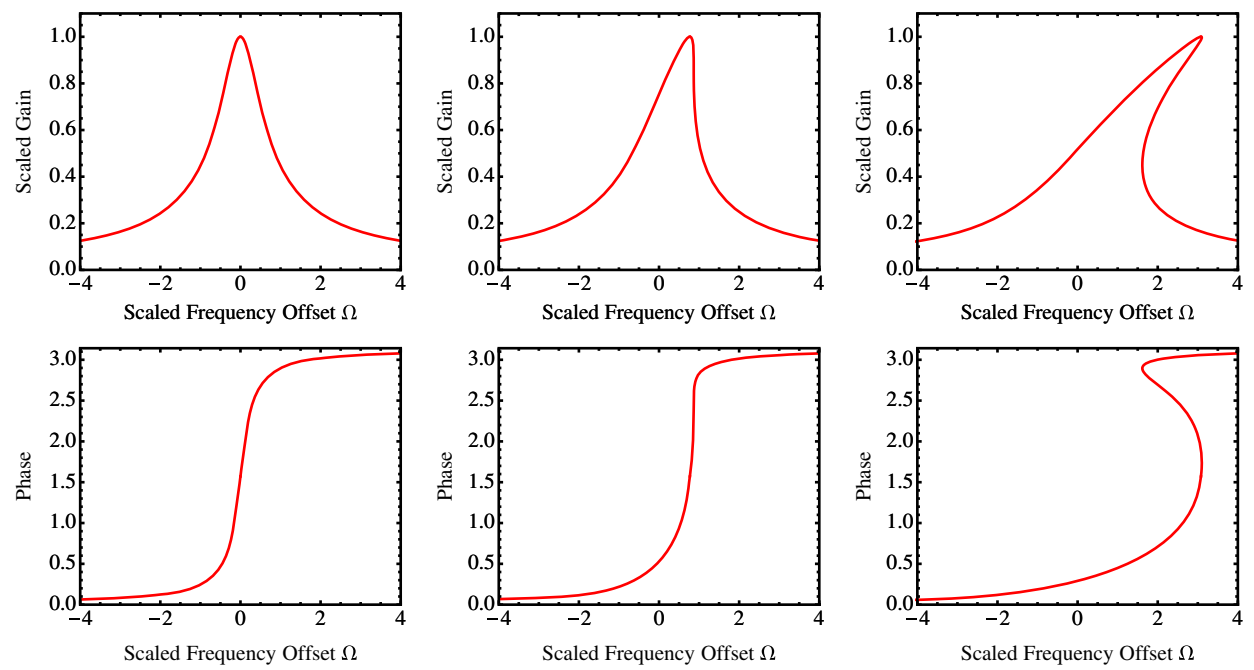

Figure 2. Scaled gain and phase response of a nonlinear resonator as a function of the scaled frequency offset $\Omega=Q\left(\omega-\omega_{0}\right) / \omega_{0}$ at different drive levels: small drive (first panels); critical drive level (middle panels); twice critical drive level (last panels).

We have extended the ideas of Greywall et al [14] in a number of directions. We follow Lax [9], Greywall et al [14], and others, in using a slow-amplitude description of the resonator dynamics. We apply secular perturbation theory to derive reduced equations of motion for the driven open-loop resonator and the closed-loop oscillator. This approach has been used to discuss a number of topics in the nonlinear dynamics of single nanomechanical resonators and resonator arrays [16-19], and the method is reviewed in [20].

The basic idea is to write the resonator motion as a slow modulation of the simple harmonic motion together with weak harmonics, using the small value of $\varepsilon=Q^{-1}$ as the expansion parameter

$$
x=\mathcal{R}\left[A \mathrm{e}^{i \omega_{0} t}\right]+\cdots .
$$

Here $A(T)=a \mathrm{e}^{i \theta}$ is the complex amplitude, with magnitude $a$ and phase $\theta$, varying on the slow time scale $T=Q^{-1} \omega_{0} t, \mathcal{R}$ denotes the real part, and the $\cdots$ denotes higher order terms in $\varepsilon$ including harmonics generated by the nonlinearity. For the driven resonator, taking $d \sim Q^{-1} D$ to reflect the small drive needed to overcome the dissipation, and $b=$ $Q^{-1} \beta$ so that the frequency shift due to the nonlinearity is comparable to the linewidth for $|A| \sim 1$, and using the standard methods of secular perturbation theory [20], leads to the equation of motion for the complex amplitude (denoting $\mathrm{d} A / \mathrm{d} T$ by $A^{\prime}$ )

$$
A^{\prime}+\frac{1}{2} A-\frac{3}{8} i \beta|A|^{2} A=-\frac{1}{2} i D \mathrm{e}^{i \Omega T},
$$

with $\omega=\omega_{0}\left(1+Q^{-1} \Omega\right)$ so that $\Omega=Q\left(\omega-\omega_{0}\right) / \omega_{0}$ is the frequency offset of the drive from the linear resonance frequency, scaled by the linewidth [21]. After transients, the solution is $A(T)=A_{0} \mathrm{e}^{i \Omega T}$ with the constant complex amplitude $A_{0}=a_{0} \mathrm{e}^{i \theta_{0}}$ satisfying

$$
\left(i \Omega+\frac{1}{2}-i \alpha\left|A_{0}\right|^{2}\right) A_{0}=-\frac{1}{2} i D
$$


where $\alpha=\frac{3}{8} \beta$, giving $x=a_{0} \cos \left(\omega t+\theta_{0}\right)$ with

$$
a_{0}^{2}=\frac{\frac{1}{4} D^{2}}{\left(\Omega-\alpha a_{0}^{2}\right)^{2}+\frac{1}{4}}, \quad \theta_{0}=\tan ^{-1} \frac{1}{2\left(\Omega-\alpha a_{0}^{2}\right)} .
$$

The expressions for the scaled gain $a_{0} / D$ and phase $\theta_{0}$ reproduce the plots in figure 2 .

The amplitude description of the resonator is based on the narrow bandwidth of the response (high $Q$ ) and weak nonlinearity so that the nonlinear frequency shifts are comparable to the linewidth. On the other hand, the feedback system (amplifier/phase-shifter combination) in the closed loop oscillator is typically strongly nonlinear (with the intensity of harmonics comparable to the fundamental) and wide bandwidth. This means that in calculating its behaviour we can neglect the slow time dependence of $A$ and calculate the fully nonlinear behaviour for a periodic input signal at the linear resonance frequency $a \cos \omega_{0} t$. This nonlinear behaviour will include up- and down-conversion of noise to frequencies near the oscillation frequency that are important to the resonator motion. The output signal from the feedback system is then projected onto the slow amplitude motion, because the resonator responds strongly only when driven near resonance. This gives the closed loop equation of motion [22]

$$
A^{\prime}+\frac{1}{2} A-i \alpha|A|^{2} A=-\frac{1}{2} i\left[g(a) \mathrm{e}^{i \Delta}+\Xi(T)\right] \mathrm{e}^{i \theta},
$$

where $g(a)$ is the amplifier gain characteristic for a harmonic signal at the oscillation frequency, $\Delta$ is the phase shift of the feedback system, and $\Xi(T)=\Xi_{\mathrm{R}}(T)+i \Xi_{\mathrm{I}}(T)$ is the complex noise given by projecting the feedback and resonator noise onto the slow time dynamics. Note that although the noise generated by the amplifier driven by the periodic signal is cyclostationary (the statistics is periodic rather than stationary) the slow noise given by the projection is stationary

$$
\left\langle\Xi_{i}(T) \Xi_{j}\left(T^{\prime}\right)\right\rangle=C_{i j}(T) \delta\left(T-T^{\prime}\right),
$$

(for $i, j$ equal to $R$ or $I$ ), as must necessarily be the case because there is no time reference for the oscillator [23]. The slow noise spectra $S_{i j}(\Omega)$ are given by the Fourier transform of the correlation functions $C_{i j}(T)$.

The stochastic amplitude equation (10) can now be solved for the diffusion of the phase $\theta$ giving the oscillator phase noise spectrum (eq. (4)) and hence the frequency precision.

The amplitude equation (10) falls into a class of systems where the noise projection method is vastly simplified [1]. These are the ones for which the dynamical variables $\mathbf{X}=\left(X_{1}, X_{2}, \ldots, X_{N-1}, \theta\right)$, here $(a, \theta)$, can be chosen such that the equations of motion are

$$
\dot{\mathbf{X}}=\mathbf{f}\left(X_{1}, X_{2}, \ldots, X_{N-1}\right),
$$

independent of $\theta$. The no-noise limit cycle is then the fixed point $X_{i}=X_{i}^{(\mathrm{s})}, i=$ $1, \ldots, N-1$, given by

$$
\mathbf{f}\left(X_{1}^{(\mathrm{s})}, X_{2}^{(\mathrm{s})}, \ldots, X_{N-1}^{(\mathrm{s})}\right)=0
$$

and then

$$
\theta=\Omega\left(X_{1}^{(\mathrm{s})}, X_{2}^{(\mathrm{s})}, \ldots, X_{N-1}^{(\mathrm{s})}\right) t .
$$

The linear stability analysis of the limit cycle is given by the constant Jacobian matrix $J_{i j}=\partial f_{i} / \partial X_{j}$, the zero-eigenvalue eigenvector of $\mathbf{J}$ is a phase shift $(0,0, \ldots, 0,1)$, 


\section{Building oscillators using nonlinear dynamics and pattern formation}

and $\mathbf{v}_{\perp}, \mathbf{v}_{n}$ are constant vectors. This means we can attempt to tune a system parameter, usually the feedback phase $\Delta$, so that $\mathbf{v}_{\perp} \cdot \mathbf{v}_{n}=0$, completely eliminating the effect of a particular noise term, represented by $\mathbf{v}_{n}$, on the frequency precision. Also, $\mathbf{v}_{\perp}$ is easily calculated as the zero-eigenvalue eigenvector of the transpose matrix $\tilde{\mathbf{J}}$. An immediate result that can be derived from this is for noise in a parameter $p$ of eq. (10), such as the feedback phase $\Delta$. From the Jacobian it is easy to show that

$$
\mathbf{v}_{\perp} \cdot \mathbf{v}_{n}=\mathrm{d} \Omega / \mathrm{d} p .
$$

Thus, to eliminate the effect of noise in the parameter $p$, the oscillator should be operated at the point where $\mathrm{d} \Omega / \mathrm{d} p=0$.

An important point is that in general the noise appearing in eq. (10) forms a ball in the complex amplitude space: even for a single noise source in the original equations of motion, $\Xi_{R}, \Xi_{I}$ will in general be imperfectly correlated due to the different averaging that occurs in projecting out the real and imaginary components. In fact, for white noise, we can choose a phase reference $\Phi_{N}$ for the noise, writing $\Xi=\mathrm{e}^{i \Phi_{N}} \bar{\Xi}$, so that $\bar{\Xi}_{\mathrm{R}}, \bar{\Xi}_{\mathrm{I}}$ are uncorrelated, giving two independent noises and correspondingly two orthogonal noise vectors $\mathbf{v}_{\text {Rn }}, \mathbf{v}_{\text {In }}$. In general, it will then not be possible to eliminate both components of the noise by setting the phase sensitivity vector $\mathbf{v}_{\perp}$ perpendicular to the noise direction by tuning the operating point. This may only be done if one of the noises $\bar{\Xi}_{R}, \bar{\Xi}_{I}$ is zero, so that the noise acts along a line in the complex amplitude space, rather than filling out a ball.

Using these ideas, we can investigate the noise reduction scheme of Greywall et al $[14,15]$. They studied the case of a saturated amplifier $g(a) \rightarrow s$, so that eq. (10) becomes

$$
A^{\prime}+\frac{1}{2} A-i \alpha|A|^{2} A=-\frac{1}{2} i\left[s \mathrm{e}^{i \Delta}+\Xi(T)\right] \mathrm{e}^{i \theta} .
$$

Writing the operating point with oscillation frequency $\Omega=\theta^{\prime}$ as $A=\bar{A}_{0} \mathrm{e}^{i \Delta} \mathrm{e}^{i \Omega T}$, eq. (16) with noise set to zero reduces to

$$
\left(i \Omega+\frac{1}{2}-i \alpha\left|\bar{A}_{0}\right|^{2}\right) \bar{A}_{0}=-\frac{1}{2} i s .
$$

Note that this is the same as eq. (8) for the driven resonator with drive level $D=s$ and $-\Delta$ playing the role of the phase shift of the response relative to the drive. For the oscillator equation however, the phase shift $\Delta$ is the control parameter, whereas the frequency $\Omega$ is determined by the dynamics, so that the $\theta_{0}(\Omega)$ curve for the resonator driven at frequency $\Omega$ is inverted to become the $\Omega(\Delta)$ curve for the oscillator. The unstable branches in the open loop configuration (the portions with $\mathrm{d} a_{0} / \mathrm{d} \Omega, \mathrm{d} \theta_{0} / \mathrm{d} \Omega<0$ ) become stabilized in the closed loop. Thus, for the saturated amplifier (and only in this limit) the oscillator operating point is directly given by the driven resonator curve.

A second feature that results from the saturated amplifier limit is that the amplifier noise in the magnitude quadrature is suppressed by the saturation. Thus, the amplifier noise appearing in eq. (10) is $\Xi \rightarrow \mathrm{e}^{i \Delta} \bar{\Xi}$ with $\bar{\Xi}_{\mathrm{R}}=0$, so that the noise acts along a line, rather than filling a ball in complex amplitude space. Equivalently, the noise can be understood as fluctuations in the feedback phase parameter. Thus, according to eq. (15), the effect of this noise on the oscillator phase noise can be eliminated by tuning the feedback phase to the 'Kenig point' where $\mathrm{d} \Omega / \mathrm{d} \Delta=0$. Since, for a saturated amplifier, $\Omega(\Delta)$ is directly given by the driven resonator response $\theta_{0}(\Omega)$ such as plotted in figure 2 , this can be achieved by tuning $\Delta$ to operate at the Duffing critical amplitude (middle panels), where the slope $\theta_{0}(\Omega)$ just becomes infinite: this is the suggestion of Greywall et al [14]. 
We can now extend the idea of Greywall et al in a number of directions. First, we can see that the condition $\mathrm{d} \Omega / \mathrm{d} \Delta=0$ can be satisfied not just at the Duffing critical point, but also for operating points giving resonator amplitudes above the critical drive level, at the two operation points giving $\mathrm{d} \theta_{0} / \mathrm{d} \Omega \rightarrow \infty$ as in the third panels of figure 2 . This can be exploited to reduce the effects of other noise sources in the system [1]. In particular, consider the direct thermomechanical noise acting on the resonator which is represented in eq. (10) by a noise ball with uncorrelated noises $\Xi_{R}, \Xi_{I}$ of equal intensities, and independent of the feedback phase $\Delta$. The component $\Xi_{\mathrm{I}}$ acting along the resonator phase acts directly on the oscillator phase, and so cannot be eliminated by tuning the operating point, but the effect can be reduced by going to larger resonator amplitudes. The component $\Xi_{R}$ along the resonator magnitude adds to the oscillator phase noise through amplitude-phase conversion: this sets the standard limitation on driving the resonator more strongly. However, this limitation can be avoided by finding operating points that eliminate amplitude-phase conversion. Quantitatively, if we write eq. (10) in the form of eq. (12) using magnitude-phase variables

$$
\begin{aligned}
& \frac{\mathrm{d} a}{\mathrm{~d} T}=f_{a}(a)+\Xi_{\mathrm{R}}, \\
& \frac{\mathrm{d} \theta}{\mathrm{d} T}=f_{\theta}(a)+a^{-1} \Xi_{\mathrm{I}},
\end{aligned}
$$

(where $\theta$ does not appear on the right-hand sides because of the phase invariance of the system) the phase sensitivity vector, given by forming the Jacobian of the linear stability analysis and finding the appropriately normalized zero-eigenvalue eigenvector of the adjoint (transpose) matrix, is

$$
\mathbf{v}_{\perp}=\left(-\frac{\partial f_{\theta} / \partial a}{\partial f_{a} / \partial a}, 1\right) .
$$

Thus, amplitude-phase conversion is eliminated when $\partial f_{\theta} / \partial a=0$. Although this condition cannot be satisfied identically with the Kenig point $\mathrm{d} \Omega / \mathrm{d} \Delta=0$ to eliminate the feedback noise, Kenig et al [1] showed that near the lower amplitude turning point of figure 2 (third panels), the two points become close for the large feedback. In fact, for large values of the drive level $s$, operating at the Kenig point leads to the following scaling of the effects of the various noise sources on the oscillator phase noise: feedback phase noise is eliminated; resonator noise in the phase quadrature $\propto s^{-2 / 3}$; and amplitude-phase conversion of resonator noise in the magnitude quadrature $\propto s^{-2}$. The major result is that the barrier for going to higher oscillator amplitudes due to the conversion of amplitude noise to phase noise by the resonator nonlinearity is removed, so that frequency performance can be significantly improved. These predictions were verified by Villanueva et al [8], as illustrated in figure 3. In particular, note the significant noise reduction around a phase shift of $170^{\circ}$ where the effects of thermomechanical noise and noise in the feedback phase and other parameters are all reduced. One noise source that is not reduced by this strategy is noise acting directly on the linear resonance frequency of the resonator, such as mass or spring constant fluctuations. These feed directly into the frequency fluctuations of the oscillator, and are not reduced by going to higher oscillation amplitudes or tuning the feedback phase. 


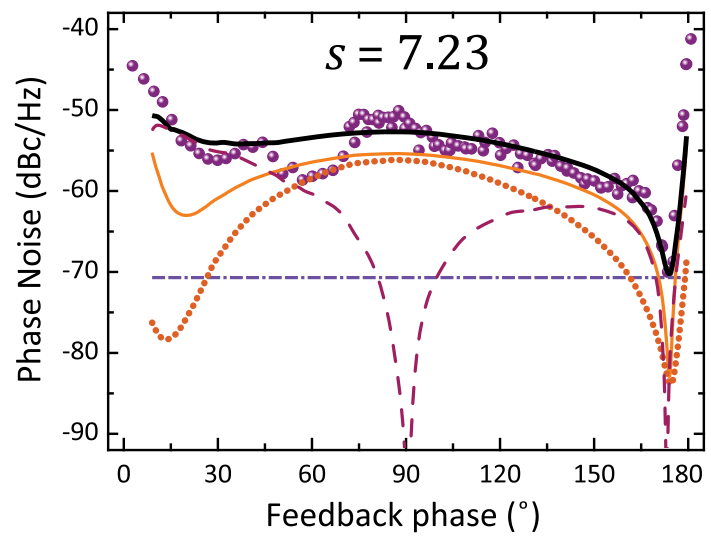

Figure 3. Oscillator phase noise as a function of feedback phase $\Delta$. The coloured lines show the predictions for different contributions to the phase noise: dashed purple line - contribution from noise in the feedback phase $\Delta$ : note the elimination at two values of $\Delta$ near $90^{\circ}$ and $180^{\circ}$; thin orange line - contribution from thermomechanical noise: note the strong suppression near $\Delta=180^{\circ}$ due to the elimination of amplitudephase conversion here; dash-dotted purple line - contribution from intrinsic frequency fluctuations, unaffected by the feedback phase; thick orange line - contributions from other parameter fluctuations; black line - prediction for total noise; purple balls experimental points. The saturation level $s=7.23$ compared with the value giving the Duffing critical amplitude $s \simeq 1.43$ (in this work a scaling with $\beta=1$ was used). For other drive levels, a discussion of how the various noise strengths were estimated, and other details, see [8] (figure extracted from figure 2 of Villanueva et al [8]).

A second extension of the idea of Greywall et al is to study the noise suppression of an unsaturated amplifier [4], such as will often be used in practical oscillators. The condition $\mathrm{d} \Omega / \mathrm{d} \Delta=0$ for eliminating the effect of noise in the phase quadrature of the feedback still applies, but $\Omega(\Delta)$ can no longer simply be read off inverting the resonator response curve $\theta_{0}(\Omega)$, and together with the amplitude of oscillation $a_{0}(\Delta)$, can be obtained by solving the full closed loop equation (10). For an unsaturated amplifier there will also in general be noise in the magnitude quadrature of the feedback giving a noise ball. It will not be possible now to eliminate the effect of both components by tuning the direction of the noise sensitivity vector, so that completely eliminating the effect of the feedback noise on the oscillator phase noise will not in general be possible. It is still potentially useful to reduce the noise to some degree by tuning $\Delta$ : the efficacy depends on the nature of the noise source and the characteristics of the amplifier. Some examples are considered in $[4,22]$.

We have also applied similar ideas to propose novel architectures for oscillators with improved frequency precision. In the parametric feedback oscillator the feedback is given by squaring the output signal of the resonator, and then applying the feedback drive parametrically as a modulation of the spring constant of the nanomechanical resonator [7]. Alternatively, we proposed using a nondegenerate parametric oscillator driven by a conventional feedback oscillator as a way of reducing the phase noise [2]. 


\section{Improving frequency precision using synchronization}

According to Winfree in his classic book [24]:

"It is often stated in research papers (it is even alleged that I proved this in 1967, which I did not) that mutual synchronization disciplines each oscillator to much improved regularity of oscillation at the common frequency."

This is an attractive idea for improving the performance of NEMS oscillators, because it is already possible to lithographically fabricate arrays of thousands of resonators [25]. Inevitably, the resonance frequencies will be slightly different due to fabrication limitations. In the synchronized state however, the interactions between the oscillators overcome this frequency dispersion and pull all the diverse oscillators to oscillate with the same frequency. The fully synchronized state is a limit cycle, and will have a deltafunction power spectrum. The question arises is the synchronized state more robust against the effects of noise causing broadening of the spectral peaks than the individual oscillators? The naive expectation might be that for independent noise sources acting on the individual oscillators, the averaging in going to the collective phase of the synchronized state would lead to a $1 / N$ reduction of the effective noise intensity for $N$ synchronized oscillators, leading to a corresponding improvement in the frequency precision. As Winfree anticipated, the result is not this simple in general [26]. In particular, as reviewed subsequently, for oscillator systems such as those based on high- $Q$ resonators where propagation effects are important, the noise reduction and frequency improvement are significantly reduced, and do not in fact scale with the total number of oscillators [3,5]. This is because the synchronized state in these systems consists of phase waves propagating from target or spiral sources, reminiscent of other oscillatory pattern forming systems such as chemical systems. The noise reduction factor turns out to be only $1 / N_{\mathrm{S}}$ with $N_{\mathrm{S}}$ the number of oscillators in a source region (defined precisely below).

We use a phase-only description of the oscillators [27]. The equations of motion are the evolution equations for the phases $\theta=\left(\theta_{1}, \theta_{2}, \ldots, \theta_{N}\right)$ of $N$ oscillators

$$
\dot{\theta}_{i}=\omega_{i}+\sum_{j \in \mathcal{N}_{i}} \Gamma\left(\theta_{j}-\theta_{i}\right)+\xi_{i}(t)
$$

with $\xi_{i}(t)$ the noise acting on the $i$ th oscillator, taken to be independent and white

$$
\left\langle\xi_{i}(t) \xi_{j}\left(t^{\prime}\right)\right\rangle=f \delta_{i j} \delta\left(t-t^{\prime}\right),
$$

and $\omega_{i}$ giving the individual oscillator frequencies, which are random variables taken from a distribution, such as a Gaussian or Lorentzian, characterized by a width $\sigma$. We shall assume nearest-neighbour coupling (denoted by the neighbourhood $\mathcal{N}_{i}$ of oscillator $i$ ) on a $d$-dimensional lattice with the coupling function $\Gamma(\Delta \theta)$, a periodic function of the phase difference $\Delta \theta$ of the oscillators. The results can be generalized to arbitrary range coupling. The coupling is dissipative if it is an odd function of phase difference, and reactive if an even function [29]: a physical realization may include both components. Reactive coupling causes differences in phase to propagate through the network of oscillators, and the synchronized state is often one of waves propagating away from one or more sources in the lattice. 


\section{Building oscillators using nonlinear dynamics and pattern formation}

For no noise and sufficiently strong coupling there will be a fully synchronized solution with all $N$ oscillators evolving at some common frequency $\Omega$ with a phase $\Theta$ of the collective limit cycle defined by

$$
\theta_{i}(t)=\theta_{i}^{(\mathrm{s})}+\Theta(t), \quad \Theta(t)=\Omega t+\text { constant }
$$

with $\theta_{i}^{(\mathrm{s})}$ given as the solution to

$$
\Omega=\omega_{i}+\sum_{j \in \mathcal{N}_{i}} \Gamma\left(\theta_{j}^{(\mathrm{s})}-\theta_{i}^{(\mathrm{s})}\right) .
$$

Noise will broaden the delta function peaks in the spectrum of the no-noise synchronized state. For small enough noise, the effect can be calculated as for the single oscillator by projecting the noise forces along the phase sensitivity vector $\mathbf{v}_{\perp}$ of the system $[3,30,31]$. This leads to diffusion of the collective phase defined by the equation of motion

$$
\dot{\Theta}=\Omega+\Xi(t), \quad\left\langle\Xi(t) \Xi\left(t^{\prime}\right)\right\rangle=F \delta\left(t-t^{\prime}\right) .
$$

Here the noise strength $F$ is given by the projection of the individual oscillator noises onto $\mathbf{v}_{\perp} \equiv \mathbf{e}_{0}^{\dagger}$, so that the noise reduction relative to the single oscillator is [3]

$$
R=\frac{F}{f}=\frac{\mathbf{e}_{0}^{\dagger} \cdot \mathbf{e}_{0}^{\dagger}}{\left(\mathbf{e}_{0}^{\dagger} \cdot \mathbf{e}_{0}\right)^{2}} .
$$

Here $\mathbf{e}_{0}=(1,1, \ldots, 1)$ is the zero-eigenvalue eigenvector of the linear stability analysis about the synchronized state defined by the Jacobian matrix $\mathbf{J}$

$$
\begin{aligned}
J_{i j} & =\Gamma^{\prime}\left(\theta_{j}^{(\mathrm{s})}-\theta_{i}^{(\mathrm{s})}\right) \quad \text { for } i, j \text { nearest neighbours, } \\
J_{i i} & =-\sum_{j \in \mathcal{N}_{i}} \Gamma^{\prime}\left(\theta_{j}^{(\mathrm{s})}-\theta_{i}^{(\mathrm{s})}\right)
\end{aligned}
$$

with other elements zero. Note that $\mathbf{e}_{0}$ is the Goldstone mode and is normalized to correspond to a uniform unit phase shift. Also, $\mathbf{v}_{\perp} \equiv \mathbf{e}_{0}^{\dagger}$ is the zero-eigenvalue adjoint eigenvector. The vectors $\mathbf{e}_{0}, \mathbf{e}_{0}^{\dagger}$ are given by solving

$$
\begin{aligned}
& \mathbf{J} \cdot \mathbf{e}_{0}=0, \\
& \mathbf{J}^{\dagger} \cdot \mathbf{e}_{0}^{\dagger}=0,
\end{aligned}
$$

with $J_{i j}^{\dagger}=J_{j i}$.

The factor $R=F / f$ gives the improvement in frequency precision of the synchronized state compared with an individual oscillator, i.e., the narrowing of the spectral peak. Note that we are treating noise perturbatively in the small noise limit and for a finite system: in this case the result is given by just the effect on the overall phase of the synchronized state, which is the zero-mode of the system. We are not considering modifications to the synchronized state due to the noise, such as changes in values of the critical disorder for synchronization, or changes in the nature of the synchronized state. In a finite system, there will be barriers to such fluctuations, and their rates will vary with the noise strength $f$ as $\mathrm{e}^{-U / f}$ with $U$ an effective barrier height depending on the states considered. These fluctuations can therefore be ignored for small enough $f$. As the number of oscillators 
tends to infinity, some barriers will become very small, and the synchronized state may be significantly changed or even eliminated by the addition of noise [32], as for phase transitions in equilibrium systems at finite temperature.

We now apply these results to specific examples of the coupling function $\Gamma$.

The standard Winfree-Kuramoto model is given by the dissipative coupling function $\Gamma(\Delta \theta)=K \sin \Delta \theta$. The properties of the system depend on the ratio of the coupling strength to the width of the disorder $K / \sigma$, and we can rescale the time variable to set $K=1$ with a rescaled $\sigma$. It is readily confirmed that for the Winfree-Kuramoto model the Jacobian is self-adjoint so that $\mathbf{e}_{0}^{\dagger}=\mathbf{e}_{0}$, and then eq. (26) gives the result $R=N^{-1}$ so that the naive averaging argument for the noise reduction and improvement in frequency precision gives the correct answer.

In the Winfree-Kuramoto model, phases relax in an overdamped way to the average phase of the neighbours. For oscillators based on high- $Q$ resonators on the other hand, one might expect oscillatory relaxation leading to propagation effects in the lattice. These effects can be included using the coupling function extended to include a reactive term of relative strength $\gamma$

$$
\Gamma(\Delta \theta)=\sin \Delta \theta+\gamma(1-\cos \Delta \theta)
$$

introduced by Sakaguchi et al [33]. The synchronized states of this model consist of waves propagating out from one or more sources $[33,34]$. Two examples from numerical simulations of a $64 \times 64$ lattice are shown in figure 4 . Either of the two sources in the figure may form, depending on initial conditions. States with multiple sources may also exist, but these will not be discussed here.

Analysing the synchronized states such as shown in figure 4 using eqs (27) and (29) for the generalized coupling function, we find the phase sensitivity vector $\mathbf{e}_{0}^{\dagger}$ to be 'localized' to a core region of $N_{\mathrm{S}}$ oscillators forming the sources [3,5]. The noise reduction ratio (eq. (26)) then gives an improvement over the single oscillator only of $1 / N_{\mathrm{S}}$. We briefly describe the calculations here, focussing on a two-dimensional lattice and using a continuum approximation. A more complete analysis based on an approximation of the interaction function on the discrete lattice rather than the continuum approximation, and results for other systems, can be found in the original papers [3,5].

We assume weak disorder (small distribution width $\sigma$ ). In this limit we expect the difference between the phases of neighbouring oscillators to be small, for all oscillators in

(a)
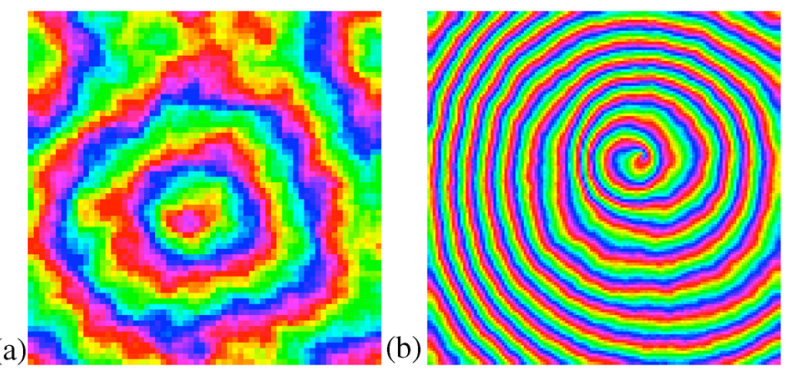

Figure 4. Synchronized states for the coupling function (eq. (29)). All the oscillators evolve with the same frequency, but with phase lags as shown by the colours giving the phases plotted from 0 to $2 \pi$ on a rainbow colour scheme. 


\section{Building oscillators using nonlinear dynamics and pattern formation}

the target state and everywhere except near the core for the spiral state. Using the interaction function (eq. (29)), setting the noise to zero, and performing a gradient expansion, eq. (21) becomes

$$
\dot{\theta}(\mathbf{r})=\omega(\mathbf{r})+\nabla^{2} \theta(\mathbf{r})+\gamma(\nabla \theta(\mathbf{r}))^{2},
$$

where $\omega(\mathbf{r})$ is a random function corresponding to $\omega_{i}$. Units of length have been set by taking the lattice constant to be unity.

We first analyse the target source, as shown in figure 4a. The Cole-Hopf transformation $\theta(\mathbf{r})=\gamma^{-1} \ln q(\mathbf{r})$ reduces eq. (30) to the linear equation

$$
\dot{q}(\mathbf{r})=\gamma \omega(\mathbf{r}) q(\mathbf{r})+\nabla^{2} q(\mathbf{r}),
$$

and the synchronized state corresponds to $\dot{q}=E q$ with $E=\gamma \Omega$ [33,34]. Equation (31) is equivalent to the Schrödinger equation (in imaginary time) for a quantum particle in a random potential $\gamma \omega(\mathbf{r})$, and the properties of the solution can be extracted from the results known for Anderson localization [35]. At long times the solution $q(\mathbf{r}, t)=q^{\max }(\mathbf{r}) \mathrm{e}^{E_{\max } t}$ corresponding to the largest eigenvalue $E_{\max }$ will dominate. This gives the synchronized state

$$
\theta^{(\mathrm{s})}(\mathbf{r})=\gamma^{-1} \ln q^{\max }(\mathbf{r}),
$$

with frequency $\Omega=E_{\max } / \gamma$. Anderson localization theory shows that $q^{\max }(\mathbf{r})$ may be chosen positive, and it has the form of an exponentially localized state centred on a region of the lattice with a potential maximum, corresponding in the oscillator problem to a concentration of larger frequency oscillators. The exponential localization of $q^{\max }(\mathbf{r})$ corresponds to a linear phase profile, defining waves propagating from a source, as shown in figure $4 \mathrm{a}$.

Within the continuum approximation, the Jacobian operator from eq. (30) is

$$
J=\nabla^{2}+2 \gamma\left(\nabla \theta^{(\mathrm{s})}\right) \cdot \nabla,
$$

giving the adjoint

$$
\hat{J}^{\dagger}=\nabla^{2}-2 \gamma \nabla \theta^{(\mathrm{s})} \cdot \nabla-2 \gamma \nabla^{2} \theta^{(\mathrm{s})} .
$$

The continuum approximation to $\mathbf{e}_{0}^{\dagger}$ is the function $e_{0}^{\dagger}(\mathbf{r})$ given by

$$
\hat{J}^{\dagger} e_{0}^{\dagger}(\mathbf{r})=0
$$

It can be verified by direct substitution that the solution is

$$
e_{0}^{\dagger}(\mathbf{r})=\mathrm{e}^{2 \gamma \theta^{(\mathrm{s})}(\mathbf{r})}=\left[q^{\max }(\mathbf{r})\right]^{2} .
$$

An example of the adjoint eigenvector $e_{0}^{\dagger}$ for a target wave source is shown in figure 5.

In the continuum approximation and using eq. (36), the noise reduction factor $R$ (eq. (26)) becomes [5]

$$
R \approx \frac{\int e_{0}^{\dagger}(\mathbf{r})^{2} \mathrm{~d} \mathbf{r}}{\left[\int e_{0}^{\dagger}(\mathbf{r}) \mathrm{d} \mathbf{r}\right]^{2}}=\frac{\int\left[q^{\max }(\mathbf{r})\right]^{4} \mathrm{~d} \mathbf{r}}{\left[\int\left[q^{\max }(\mathbf{r})\right]^{2} \mathrm{~d} \mathbf{r}\right]^{2}}
$$

This equation directly relates the improvement in frequency precision to the solution of the linear Anderson problem (eq. (31)). The last expression in eq. (37) is the inverse participation ratio $p^{-1}$ of the function $q^{\max }(\mathbf{r})$ of the linear localization problem. Thus, we find 
(a)

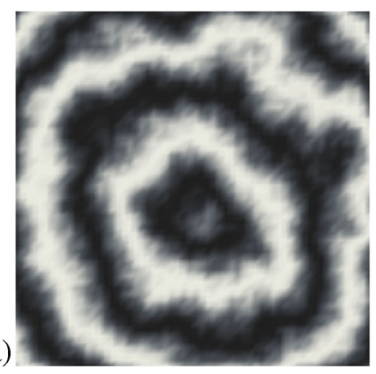

(b)

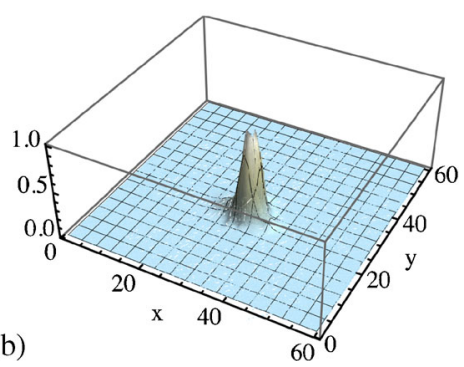

Figure 5. Target waves and source for a realization of a $60 \times 60$ two-dimensional lattice of oscillators with $\gamma=1, \sigma=2$. (a) Grey scale plot of $\cos \theta_{i}^{(\mathrm{s})}$ giving a snapshot of the waves emanating from the source; (b) zero-eigenvalue adjoint eigenvector $\mathbf{e}_{0}^{\dagger}$ showing the effective size of the source of the waves.

that the noise reduction factor is given by the size of the number of oscillators $N_{\mathrm{S}}=p$ acting as the source of the waves, rather than by the total number of oscillators, giving an improvement in frequency stability that is significantly worse for a large number of entrained oscillators. The size of the source is defined precisely in terms of the participation ratio of the maximum energy localized state of the corresponding Anderson problem. For the system in figure 5, $p \simeq 9.36$; in this example, the frequency precision would not be improved by increasing the number of oscillators beyond about ten. From eqs (37) and (31) it is apparent that the source size and noise reduction factor $R$ depend on the parameters of the model only through the product $\gamma \sigma$, for the approximations used. The size of the source will increase with decreasing disorder strength $\sigma$ and reactive constant $\gamma$.

Figure $4 \mathrm{~b}$ shows a spiral source. The spiral source is not completely captured by the approximation of small phase differences used for the target sources, because at the core, neighbouring oscillators necessarily have large phase differences. The spirals are not predicted by the mapping onto the linear Anderson problem, and we must proceed by a different route to understand the properties [5]. Unlike the target source, spiral sources exist even in the completely uniform lattice, and it is simplest to analyse their behaviour in this limit. Without loss of generality, we then set the common oscillator frequency to zero. We expect that small disorder may give small quantitative changes to the expressions, but will not change the main conclusions.

Far away from the core of the spiral the phase differences between adjacent oscillators do remain small, and we may continue to use the continuum approximation. The synchronized state in this region is $\theta^{(\mathrm{s})}=\gamma^{-1} \ln q^{(\mathrm{s})}$ with $q^{(\mathrm{s})}(\mathbf{r})$ the solution to

$$
\nabla^{2} q^{(\mathrm{s})}=k^{2} q^{(\mathrm{s})}, \quad k=\sqrt{\Omega \gamma} .
$$

Solving this equation for a single armed spiral centred at the origin in polar coordinates $\mathbf{r} \rightarrow(\rho, \varphi)$ gives

$$
\theta^{(\mathrm{s})}(\rho, \varphi)=\varphi+\gamma^{-1} \ln K_{i \gamma}(k \rho),
$$

where $K_{\alpha}(z)$ is the modified Bessel function of the second kind. The value of $k$ must be real and positive, which means the spiral waves are outgoing from the centre; this 
condition requires $\Omega \gamma>0$, and so the frequency of the spiral is greater (less) than the frequencies of the individual oscillators for $\gamma>0(\gamma<0)$. For definiteness and without loss of generality, we take $\gamma>0$ and so $\Omega>0$. In a finite system, there will be corrections to eq. (39) within a distance $\sim k^{-1}$ from the boundaries.

The zero-eigenvalue adjoint eigenvector can again be found analytically. It can be verified by direct substitution that the solution to eq. (34) with $\theta^{\text {(s) }}$ given by eq. (39) is the purely radial function

$$
e_{0}^{\dagger}(\rho)=\mathrm{e}^{2 \gamma \psi(\rho)},
$$

with $\psi(\rho)=\gamma^{-1} \ln K_{i \gamma}(k \rho)$ the radial part of the spiral solution (39). The noise reduction ratio (37) becomes

$$
R=k^{2} F(\gamma),
$$

where $F(\gamma)$ is plotted in figure 6 (it can be expressed in terms of a Meijer's G-function). $R$ is given by $k^{2}$ multiplied by an $O(1)$ slowly varying function of $\gamma$, and $k^{-1}$ sets the length scale of the spiral and defines the core size. In a finite system of $N$ oscillators, as the adjoint eigenvector decays as $\mathrm{e}^{-2 k \rho} / \rho$ for large $\rho$, there will be corrections from the boundaries when $k \sqrt{N}$ is insufficiently large. The important conclusion of this calculation is that, because $k=\sqrt{\Omega \gamma}$ is independent of $N$ for large systems, the noise reduction ratio $R$ is again some constant that does not increase with $N$ for large $N$. This was verified numerically in [5] over the range $0.29<\gamma<0.43$.

To calculate $k(\gamma), \Omega(\gamma)$ and so $R(\gamma)$ requires matching to the core region of the spiral where the continuum approximation breaks down. Following Hagan [36], who investigated spiral formation in continuum amplitude-phase equations for collective oscillations, we anticipate this matching to lead to an expression of the form $k(\gamma)=$ $2 \exp (-(\pi / 2 \gamma)+\alpha)$, where the constant $\alpha$ is determined by the core structure. The value of $\alpha \simeq 0.84$ is obtained by fitting $\Omega=k^{2} / \gamma$ to numerical results for the spiral frequency (see [5] for details). Together with figure 6, this provides a complete prescription for determining the effective noise reduction and improvement in the frequency precision for a spiral synchronized state.

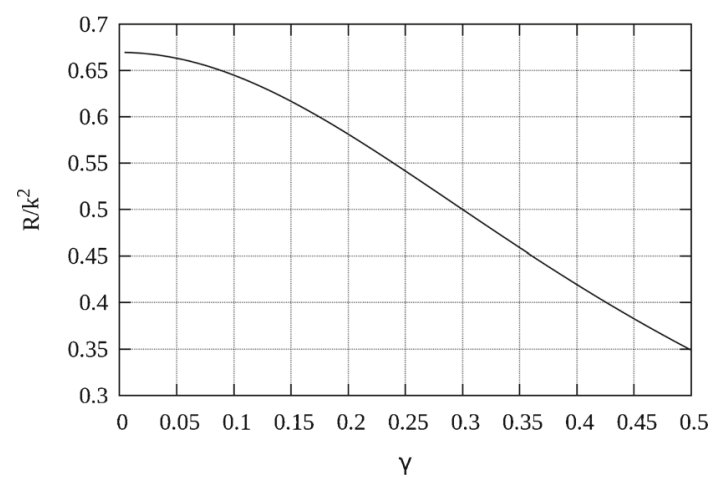

Figure 6. Plot of $R / k^{2}$ derived from the analytic continuum approximation, with $R$ the relative frequency precision and $k=\sqrt{\Omega \gamma}$ with $\Omega$ the spiral frequency, as a function of the reactive coupling parameter $\gamma$ (figure reproduced from [5]). 


\section{Conclusions}

For centuries, clocks and frequency references have been based on resonators with drive levels restricted to the linear regime. In the work reviewed in this paper, we have studied exploiting nonlinearity to improve performance. We have shown how the detrimental effects of nonlinearity in a single resonator converting amplitude to phase noise can be mitigated, whilst at the same time using the resonator nonlinearity to improve performance by eliminating the effects of feedback drive noise and other noise. In addition, we have proposed novel oscillator architectures, and have analysed how the synchronization of many oscillators can further improve the performance. Our work exemplifies the importance of nonlinear dynamics and pattern formation to modern technology.

\section{Acknowledgements}

This work would not have been possible without the close collaboration with the Caltech nanomechanical experimental group and the authors acknowledge in particular the important contributions of Michael Roukes, Rassul Karabalin, Matt Matheny and Guillermo Villanueva. Ron Lifshitz, Jeff Moehlis and Kurt Wiesenfeld also contributed to the theoretical ideas. The material is based upon work supported by the National Science Foundation under Grant No. DMR-1003337 and DARPA through the DEFYS programme. JMAA was supported by the Master and Fellows of Corpus Christi College and the California Institute of Technology.

\section{References}

[1] E Kenig, M C Cross, L G Villanueva, R B Karabalin, M H Matheny, R Lifshitz and M L Roukes, Phys. Rev. E 86, 056207 (2012)

[2] E Kenig, M C Cross, R Lifshitz, R B Karabalin, L G Villanueva, M H Matheny and M L Roukes, Phys. Rev. Lett. 108, 264102 (2012)

[3] M C Cross, Phys. Rev. E 85, 046214 (2012)

[4] E Kenig, M C Cross, J Moehlis and K Wiesenfeld, Phys. Rev. E 88, 062922 (2013)

[5] J-M A Allen and M C Cross, Phys. Rev. E 87, 052902 (2013)

[6] E Kenig and M C Cross, Phys. Rev. E 89, 042901 (2014)

[7] L G Villanueva, R B Karabalin, E Matheny, M H andKenig, M C Cross and M L Roukes, Nano Lett. 11, 5054 (2011)

[8] L G Villanueva, E Kenig, R B Karabalin, M H Matheny, R Lifshitz, M C Cross and M L Roukes, Phys. Rev. Lett. 110, 177208 (2013)

[9] M Lax, Phys. Rev. 160, 290 (1967)

[10] F X Kaertner, Int. J. Circuit Theory Appl. 18, 485 (1990)

[11] A Demir, A Mehrotra and J Roychowdhury, IEEE Trans. Circuits Syst. 47, 655 (2000)

[12] We will only discuss white noise sources here. For special features of $1 / f$ noise sources, refer Kenig and Cross [6]

[13] For some situations, for example, parametrically driven resonators, we also need to include a nonlinear damping term

[14] D S Greywall, B Yurke, P A Busch, A N Pargellis and R L Willett, Phys. Rev. Lett. 72, 2992 (1994)

[15] B Yurke, D S Greywall, A N Pargellis and P A Busch, Phys. Rev. A 51, 4211 (1995) 


\section{Building oscillators using nonlinear dynamics and pattern formation}

[16] R Lifshitz and M C Cross, Phys. Rev. B 67, 134302 (2003)

[17] M C Cross, A Zumdieck, R Lifshitz and J L Rogers, Phys. Rev. Lett. 93, 224101 (2004)

[18] M C Cross, J L Rogers, R Lifshitz and A Zumdieck, Phys. Rev. E 73, 036205 (2006)

[19] R B Karabalin, M C Cross and M L Roukes, Phys. Rev. B 79, 165309 (2009)

[20] R Lifshitz and M C Cross, in: Review of nonlinear dynamics and complexity edited by $\mathrm{H} \mathrm{G}$ Schuster (Wiley, Meinheim, 2008) Vol. 1, pp. 1-52

[21] There are many different conventions for introducing the small parameter $\varepsilon$, for example sometimes a factor of $\varepsilon$ is introduced in the leading-order term in eq. (6). The resulting equation is always, of course, the same eq. (7)

[22] E Kenig and M C Cross, Frequency precision of oscillators based on high- $Q$ resonators (2014), preprint

[23] Note that this is only true with the noise in eq. (10) appearing with the phase factor $\mathrm{e}^{i \theta}$. For a proof that this is the case, see [22]

[24] A Winfree, The geometry of biological time (Springer, New York, 1984)

[25] I Bargatin, E B Myers, J S Aldridge, C Marcoux, P Brianceau, L Duraffourg, E Colinet, S Hentz, P Andreucci and M L Roukes, Nano Lett. 12, 1269 (2012)

[26] N Masuda, Y Kawamura and H Kori, New J. Phys. 12, 093007 (2010)

[27] For an example of the reduction of a complex amplitude description to a phase only description, see Zumdieck et al [28]

[28] M C Cross, J L Rogers, R Lifshitz and A Zumdieck, Phys. Rev. E 73, 036205 (2006)

[29] D Topaj and A Pikovsky, Physica D 170, 119 (2002)

[30] Y Kawamura, H Nakao, K Arai, H Kori and Y Kuramoto, Phys. Rev. Lett. 101, 024101 (2008)

[31] H Kori, Y Kawamura, H Nakao, K Arai and Y Kuramoto, Phys. Rev. E 80, 036207 (2009)

[32] J A Acebron, L L Bonilla, C J P Vicente, F Ritort and R Spigler, Rev. Mod. Phys. 77, 137 (2005)

[33] H Sakaguchi, S Shinomoto and Y Kuramoto, Prog. Theor. Phys. 79, 1069 (1988)

[34] B Blasius and R Tonjes, Phys. Rev. Lett. 95, 084101 (2005)

[35] P W Anderson, Phys. Rev. 109, 1492 (1958)

[36] P S Hagan, SIAM J. Appl. Math. 42(4), 762 (1982) 